Gefässchirurgie 2014 • 19:510-512

DOI 10.1007/s00772-014-1315-0

Online publiziert: 20. Oktober 2014

(c) Springer-Verlag Berlin Heidelberg 2014

H.H. Eckstein ${ }^{1}$ I. Flessenkämper ${ }^{2} \cdot$ H. Wenk ${ }^{3}$ - E.S. Debus ${ }^{4}$

${ }^{1}$ Klinik für Vaskuläre und Endovaskuläre Chirurgie und Münchener Aorten Centrum (MAC), Klinikum rechts der Isar der Technischen Universität München

${ }^{2}$ HELIOS Klinikum Emil vom Behring, Berlin

${ }^{3}$ Zentrum für Gefäßmedizin, Klinikum Bremen-Nord

${ }^{4}$ Universitätsklinikum Hamburg-Eppendorf

\title{
Ultraschallscreening auf abdominale Aortenaneurysmen
}

ne ausführliche Stellungnahme abgegeben hat. Im Mai 2014 wurde ein aktualisierter Berichtsplan inkl. der oben genannten DGG-Stellungnahme auf der Homepage des IQWIG veröffentlicht [4]. Auf Basis dieses Berichtplans wird derzeit ein Vorbericht erarbeitet, der ebenfalls zur Anhörung noch im Jahr 2014 veröffentlicht werden wird. Dieser Vorbericht wird auch einem externen Review unterzogen werden. Im Anschluss an diesen Prozess wird das IQWIG einen Abschlussbericht erstellen, der dann dem G-BA vorgelegt und ebenfalls einige Wochen später auf der IQWIG-Homepage veröffentlicht werden wird.

Warum diese komplizierten Detailinformationen in einem Editorial der Gefässchirurgie? Nun, das AAA-Screening ist in greifbare Nähe gerückt! Der Gang durch die Institutionen ist beschlossen und auf einem guten und übrigens auch methodisch sehr transparenten Weg. Das ist eine überaus erfreuliche Entwicklung und die DGG wird sich so aktiv und konstruktiv wie irgend möglich in diesen Prozess einschalten. Deshalb auch ein Schwerpunktheft der Gefässchirurgie zum Ultraschallscreening auf abdominale Aortenaneurysmen (AAA). Wir danken an dieser Stelle allen Autoren, für ihre spontanen Zusagen, die zugedachten Artikel zu verfassen. Ganz besonders wichtig ist aus unserer Sicht auch die ausführliche Darstellung der bereits etablierten Screeningprogramme in England, Schwe- den, den USA sowie eines regionalen Programms in Polen. Unser Dank gilt auch der Deutschen Gesellschaft für Angiologie (DGA), die ein nationales AAA-Screening-Programm vorbehaltlos und aktiv unterstützt.

\section{1) Das AAA-Screening ist \\ in greifbare Nähe gerückt!}

Im Gast-Editorial der DGA wird richtigerweise an die 10 klassischen Kriterien für ein erfolgreiches Screeningprogramm der Weltgesundheitsorganisation (WHO) aus dem Jahr 1968 erinnert, die beim AAA-Screening nahezu 100\%ig erfüllt werden [5] (• Tab. 1). Diese Kriterien wurden in einem aktuellen Artikel des Bulletin of the World Health Organization präzisiert und aktualisiert [6]. Die Gegenüberstellung dieser aktualisierten Anforderungen und deren möglicher Übertragbarkeit auf ein AAA-Screening in Deutschland zeigen, dass im Detail noch viele logistische Aufgaben bei der konkreten Realisation eines landesweiten AAA-Screening-Programms zu bewältigen sind. Sehr kritisch muss auch die bisher nur freiwillige Qualitätskontrolle/-sicherung der AAA-Operationen in Deutschland gesehen werden. Sehr wahrscheinlich ist ein AAA-Screening nur dann zu verantworten, wenn gleichzeitig alles getan wird, um die operative Therapie des AAA so sicher wie irgend möglich 


\begin{tabular}{|c|c|}
\hline Revised WHO criteria for screening programmes [5] & Übertragbarkeit auf ein AAA-Screening in Deutschland \\
\hline $\begin{array}{l}\text { The screening programme should respond to a recognized } \\
\text { need }\end{array}$ & $\begin{array}{l}\text { Mehr als } 2000 \text { dokumentierte AAA-Rupturen pro Jahr, hohe Dunkelziffer, geschätzt ins- } \\
\text { gesamt } 6000 \text { bis } 8000 \text { AAA-Rupturen pro Jahr }\end{array}$ \\
\hline The objectives of screening should be defined at the outset & Senkung der AAA-assoziierten Mortalität \\
\hline There should be a defined target population & $\begin{array}{l}\text { Ältere Menschen (insbesondere Männer), familiäre AAA-Belastung, Patient(inn)en mit } \\
\text { Nikotinabusus und/oder kardiovaskulären Begleiterkrankungen }\end{array}$ \\
\hline $\begin{array}{l}\text { There should be scientific evidence of screening programme } \\
\text { effectiveness }\end{array}$ & $\begin{array}{l}\text { Metaanalysen, systematische Reviews und Health Technology Assessments (HTA-Be- } \\
\text { richte) multipler randomisierter Studien }\end{array}$ \\
\hline $\begin{array}{l}\text { The programme should integrate education, testing, clinical } \\
\text { services and programme management }\end{array}$ & $\begin{array}{l}\text { Noch zu tun: Trainingsprogramme und Zertifizierungen für Screeningeinheiten und/ } \\
\text { oder niedergelassene Ärztinnnen/Ärzte. Verpflichtende Dokumentation und zentrale } \\
\text { Auswertung der Ultraschallbefunde (Vorlage: Disease-Management-Programme?) }\end{array}$ \\
\hline \multirow[t]{2}{*}{$\begin{array}{l}\text { There should be quality assurance, with mechanisms to } \\
\text { minimize potential risks of screening }\end{array}$} & $\begin{array}{l}\text { Noch zu tun: verpflichtende Qualitätssicherung (QS) der AAA-Operationen (vergleich- } \\
\text { bar zu Karotis-TEA bzw. Karotis-Stent). Basis: aktuelles QS-Programm zum AAA der DGG }\end{array}$ \\
\hline & Gegebenenfalls weitere Zentralisierung der operativen Therapie des AAA \\
\hline $\begin{array}{l}\text { The programme should ensure informed choice, confidentiality } \\
\text { and respect for autonomy }\end{array}$ & $\begin{array}{l}\text { Noch zu tun: Patientenaufklärung über Krankheitsbild, Ursachen, Verlauf und Therapie- } \\
\text { optionen }\end{array}$ \\
\hline $\begin{array}{l}\text { The programme should promote equity and access to scree- } \\
\text { ning for the entire target population }\end{array}$ & $\begin{array}{l}\text { Noch zu tun: Organisation des AAA-Screenings über Gefäßzentren und niedergelasse- } \\
\text { ne Fachärzt(inn)en (Allgemeinmedizin, kardiovaskuläre Fächer etc.) }\end{array}$ \\
\hline Programme evaluation should be planned from the outset & $\begin{array}{l}\text { Noch zu tun: Kooperation u. a. von Fachgesellschaften, Kassenärztlichen Vereinigungen, } \\
\text { Krankenhäusern u. v. a. }\end{array}$ \\
\hline The overall benefits of screening should outweigh the harm & $\begin{array}{l}\text { Hochsignifikante Reduktion der AAA-assoziierten Mortalität in randomisierten Studien, } \\
\text { Metaanalysen, systematischen Reviews und HTA-Berichten }\end{array}$ \\
\hline
\end{tabular}

anzubieten. Diese Überlegungen beinhalten dann auch die in Deutschland bislang unzureichende Analyse des Volume-Outcome-Zusammenhangs bei der Behandlung des AAA. Die Daten des AAA-Qualitätssicherungsprogramms der DGG (siehe Trenner et al. in diesem Heft) deuten darauf hin, dass zumindest bei der offenen AAA-Therapie höhere Fallzahlen zu besseren Ergebnissen führen. Derzeit wird dieses Ergebnis in Zusammenarbeit mit dem Statistischen Bundesamt auf Bundesebene überprüft. Eine AAABehandlung in mehr als 500 Krankenhäusern ist mittelfristig sicherlich nicht zu rechtfertigen [6].

Wir hoffen sehr, dass vielleicht in 2015/2016 auch in Deutschland ein durch die gesetzlichen Kassen mitgetragenes AAA-Screening eingeführt werden wird. Die Evidenz liegt auf dem Tisch, die Machbarkeit erscheint gegeben, packen wir's also an. Für die Gefäßchirurgie ergibt sich die wirklich einmalige Chance, in der allgemeinen Krankheitsvorsorge und Krankenversorgung eine Indikatorleistung zu erbringen, die uns von keinem anderen Fach streitig gemacht werden kann. Ein kassenfinanziertes AAAScreening würde helfen, die Marke "Gefäßchirurgie" in der öffentlichen Wahrnehmung signifikant nach vorn zu brin- gen und uns damit auch aus der manchmal geliebten aber letztendlich doch unbefriedigenden Nische des Spezialistentums sichtbarer hervortreten lassen. Vielleicht kann dieses Schwerpunktheft dazu einen Beitrag leisten.

In diesem Zusammenhang möchten wir Sie sehr herzlich zum Münchner AAA-Screening-Symposium am 4. Dezember 2014 einladen. In dieser gemeinsamen Veranstaltung der DGG und der MUNICH AORTIC \& CAROTID CONFERENCE (MAC) wollen wir zusammen mit Vertretern des G-BA, der Krankenkassen, der Politik und weiterer Entscheidungsebenen über die konkreten Umsetzungsmöglichkeiten eines AAA-Screenings in Deutschland diskutieren. Hierzu werden wir auch auf internationale Erfahrungen zurückgreifen können, da wichtige Repräsentanten laufender Screeningprogramme in England, Schweden etc. ihre Teilnahme und Unterstützung bereits zugesagt haben [8].

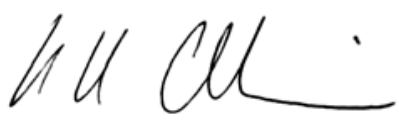

Hans-Henning Eckstein

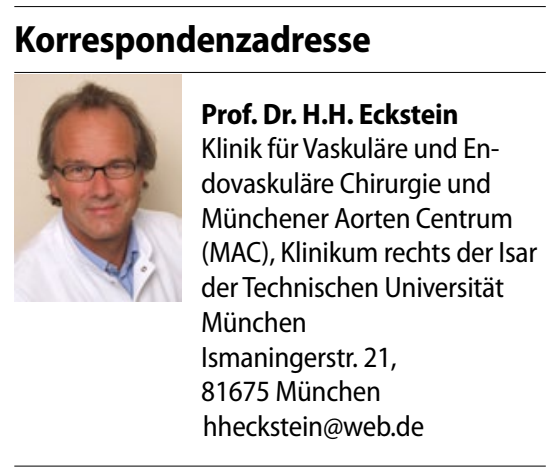

Interessenkonflikt. H.H. Eckstein, I. Flessenkämper, H. Wenk und E. S. Debus geben an, dass kein Interessenkonflikt besteht.

\section{Literatur}

1. Beschluss des Gemeinsamen Bundesausschusses über die Einleitung eines Beratungsverfahrens zur Prüfung gemäß § 135 Abs. 1 in Verbindung mit § 25 SGB V der Methode "Ultraschall-Screening auf abdominale Bauchaortenaneurysmen" vom 18. April 2013. https://www.g-ba.de/downloads/39-261-1734/2013-04-18_Antragsannahme_ Screening-Bauchaortenaneurysmen.pdf

2. Bundesministerium für Gesundheit (2013) Bekanntmachung des Gemeinsamen Bundesausschusses über weitere Beratungsthemen zur Überprüfunggemäß § 135 Absatz 1 des Fünften Buches Sozialgesetzbuch (SGBV) in Verbindung mit § 25 SGB V: Prüfung des Ultraschall-Screenings auf Bauchaortenaneurysmen, veröffentlicht am Donnerstag, 14. November 2013 BAnz AT 14.11.2013 B6 
3. Antrag der Patientenvertretung nach $\S 140 \mathrm{f}$. SGB V Ultraschall-Screening auf abdominale Bauchaortenaneurysmen 2013. https://www.g-ba.de/ downloads/40-268-2565/1-2013-04-18_Antrag_ PatV_U-Screening-BAA.pdf

4. https://www.iqwig.de/download/S13-04_Berichtsplan_Ultraschall-Screening-auf-Bauchaortenaneurysmen.pdf

5. Wilson JMG, Jungner G. Principles and practice of screening for disease Geneva:WHO; 1968. Available from: http://www.who.int/bulletin/volumes/ 86/4/07-050112BP.pdf

6. Andermann $A$, Blancquaert I, Beauchamp $S$, Déryc $V$ (2008) Revisiting Wilson and Jungner in the genomic age: a review of screening criteria over the past 40 years. Bull World Health Organ 86(4):317319

7. Eckstein HH (2014) Operieren wir zu viel oder operieren zu viele? Gefässchirurgie 19(5):397-398

8. http://www.mac-conference.com/de/Home/AAAScreening-Symposium.html

\section{Antibiotikatoleranz langsam wachsender Bakterien}

Infektionsbiologen der Universität Basel konnten zeigen, dass Bakterien einer Art auch bei Ansiedlung im selben Gewebe eine stark unterschiedliche Antibiotikatoleranz aufweisen und vor allem langsam wachsende Keime den Erfolg von Antibiotikatherapien gefährden.

Bereits aus früheren Studien weiß man, dass im Laborversuch gleiche Bakterien trotz identischer Bedingungen sehr unterschiedlich schnell wachsen können. Unklar war bislang, ob sich Bakteriengesellschaften auch im infizierten Wirt so unterschiedlich verhalten. Den Wissenschaftlern des Biozentrums der Universität Basel gelang es nun, mithilfe fluoreszierender Proteine die Vermehrung einzelner Salmonellen in infizierten Geweben von Mäusen zu messen. Es zeigte sich, dass ein Teil der Salmonellen sehr schnell wuchs. Die meisten Bakterien jedoch gelangten in Geweberegionen mit wenigen Nährstoffen, in denen sie nur langsam wachsen konnten. Dieses unterschiedliche Wachstumsverhalten wirkte sich deutlich auf den Therapieerfolg mit Antibiotika aus. Eine Behandlung der infizierten Mäuse verbesserte sehr schnell die Krankheitssymptome, doch selbst nach fünf Tagen Therapie waren noch immer Erreger nachweisbar, die einen Rückfall verursachen könnten. Während die schnell wachsenden Bakterien zu etwa $90 \%$ schon mit der ersten Antibiotikadosis abgetötet wurden, überlebten die langsam wachsenden Erreger deutlich länger. Der Behandlungserfolg hing folglich entscheidend von der Vermehrungsrate der Bakterien ab.

Bisher vermuteten viele Forscher, dass neben resistenten Bakterien vor allem Keime, die sich in einer Art „Schlafzustand“ befinden und sich nicht teilen, Antibiotikabehandlungen auch über lange Zeiträume hinweg überleben können. Dagegen belegen die neuen Erkenntnisse, dass die langsam wachsenden Bakterien ein größeres Problem für die Therapie darstellen. Sie tolerieren Antibiotika zwar etwas schlechter als schlafende Keime, sind aber viel zahlreicher und können ihr Wachstum jederzeit wieder steigern. Ein besseres Verständnis dieser Bakterien könnte in Zukunft eine gezielte Antibiotikatherapie über kurze Behandlungszeiträume hinweg ermöglichen. Das ist vor allem bei Infektionen inter- essant, bei denen Patienten ihre Medikamente über viele Tage und Wochen einnehmen müssen, um einen Rückfall zu verhindern.

Literatur:

Claudi B, Spröte P, Chirkova A et al (2014)

Phenotypic Variation of Salmonella in Host

Tissues Delays Eradication by Antimicrobial

Chemotherapy. Cell 158:722-33

Quelle:

Universität Basel, www.unibas.ch 